\title{
Effects of Persister Formation on Bacterial Response to Dosing
}

\author{
N. G. Cogan*
}

*Mathematics Department Tulane University New Orleans, LA. 70118 e-mail: cogan@math.tulane.edu FAX: 5048655063 


\begin{abstract}
Almost all moist surfaces are colonized by microbial biofilms. Biofilms are implicated in cross-contamination of food products, biofouling and various human infections such as dental cavities, ulcerative colitis and chronic respiratory infections. The recalcitrance of biofilms to typical antibiotic and antimicrobial treatments is one focus of current investigations. Neither reaction-diffusion limitation nor heterogeneities in growth-rate explain the observed tolerance. Another hypothesis is that specialized 'persister' cells, which are extremely tolerant of antimicrobials, are the source of resistance.

In this investigation, we describe the formation of 'persister' cells which neither grow nor die in the presence of antibiotics. We propose that these cells are of a different phenotype whose expression is regulated by the growth rate and the antibiotic concentration. Based on several experiments describing the dynamics of persister cells, we introduce a mathematical model that is used to describes the effect of a periodic dosing regiment. Results from our analysis indicate that the relative dose/withdrawal times are important in determining the effectiveness of such a treatment. A reduced model is also introduced and the similar behavior is demonstrated analytically.
\end{abstract}

Keywords: Persister, tolerance, biofilm, dosing, resistance

\title{
1 Introduction
}

It has been estimated that $99 \%$ of all bacteria live in structured communities termed biofilms [2]. Recently the US National Institutes of Health announced that, "Biofilms are medically important, accounting for over $80 \%$ of microbial infections in the body". Understanding resistance mechanisms for bacterial biofilms is of paramount importance in treating bacterial infections $[7,8,21,1,14,20]$. There are several hypotheses concerning resistance mechanisms which can be placed into three broad categories: transport limitation, physiological tolerance and phenotypic resistance.

Biofilm structure can prevent an applied antimicrobial agent from reaching the entire bacterial population by mechanisms such as a neutralizing reaction with components of the biofilm $[29,10]$, synthesis of an antimicrobial degrading enzyme [16, 3] and adsorption of the antimicrobial by the exo-polymeric substance (EPS) [19]. There have been several mathematical models of biofilm disinfection that include diffusion and various antimicrobialdegrading reactions $[25,12,6]$. These studies indicate that if the reaction is catalytic and does not degrade the neutralizing agent, bacteria deep within the biofilm are not exposed to the antimicrobial agent. Otherwise, even though lowered diffusion and degrading reactions slow the penetration, the antimicrobial will eventually penetrate the entire biofilm. Unless there are other protective mechanisms at work the bacteria would eventually be eradicated.

Because most biocides and antibiotics are more effective at killing respiring bacteria than non-respiring bacteria [7], disinfection will be spatially dependent. In particular, there will be regions within the biofilm where nutrient is not available. The bacteria in these regions will exhibit decreased respiration leading to decreased susceptibility to disinfection. This protective mechanism is termed physiological resistance. Analysis of mathematical models predict that bacteria on the surface of the biofilm are killed before those deep within the 
biofilm [6, 23] . Since the nutrient penetrates further as the bacteria are killed, constant exposure to antimicrobials will eventually eradicate the bacteria. Since long-term exposure to antimicrobials does not lead to complete eradication of bacteria $[28,17,8]$ other resistance mechanisms must be explored.

The focus of this paper is on the mechanism of phenotypic resistance. Because transport limitation and physiological tolerance cannot explain the typical bi-phasic nature of survival data, tolerance due to phenotypic variation is currently being investigated [27, 21]. It has been shown that quorum sensing mechanisms, known to be linked with biofilm formation [9], may also be linked to antibiotic resistance [22, 13, 27]. Quorum sensing is involved in upregulation of multi-drug efflux pumps [4], although this may not play a role in the antibioticresistant phenotype [18]. Variations in the maximum specific growth rates may result in varying susceptibility of bacteria [27]. A novel explanation of the bi-phasic disinfection curves is the existence of 'persister' cells which are extremely tolerant of antibiotics. The physiology of such cells is not well understood, although their existence has been demonstrated [11, 17, 21]. As yet, there is no consensus as to what phenotypic variations are primarily responsible for biofilm tolerance [21].

Here, we incorporate current biological observations into a mathematical model of bacterial tolerance. This model is used to explore optimal dosing strategies. Although the model applies to spatially uniform populations of bacteria, we view this as a first step towards a more realistic model in a biofilm setting. We first summarize the experiments that motivate the model, then describe our assumptions and consequent model. Several simulations used to estimate parameters and explore dosing protocols will be described. Finally, we introduce a simplified model which can be treated analytically.

\section{Planktonic Experiments}

Bacterial tolerance to antibiotics has been well established, but the specific mechanisms are still being investigated. In [11] the tolerance of both planktonic and biofilm cultures of $B$. cepecia bacteria to ciprofloxacin and ceftazidime was compared. Bacterial populations were grown in rotary shakers (planktonic) and polycarbonate membranes (biofilm). At given times, samples of comparable number were taken, diluted and exposed to the antibiotics for one hour. By sampling at different times, resistance is linked to the growth stage. That is, the untreated population showed logistic growth with little or no lag stage, followed by exponential growth and then a drop in the growth rate due to nutrient limitation. Both planktonic and biofilm bacteria showed drastic increase in tolerance to both antibiotics during during exponential growth. Thus, the growth rate/phase of the culture plays an important role in determining antibiotic susceptibility. In this study the planktonic bacteria are always more susceptible to biofilm bacteria at equivalent stages of growth although this result has been contradicted [26]. Because the biofilm cultures were disrupted and suspended before treating them, the increased resistance is not from any physical barrier. Instead, tolerance results from physiological and phenotypic variations.

In another investigation similar experiments were performed on planktonic populations of E. coli, P. aeruginosa and S. aureus [17]. The focus of this investigation is on the generation of persisters, cells which neither grow nor die in the presence of antibiotics. Cultures of 


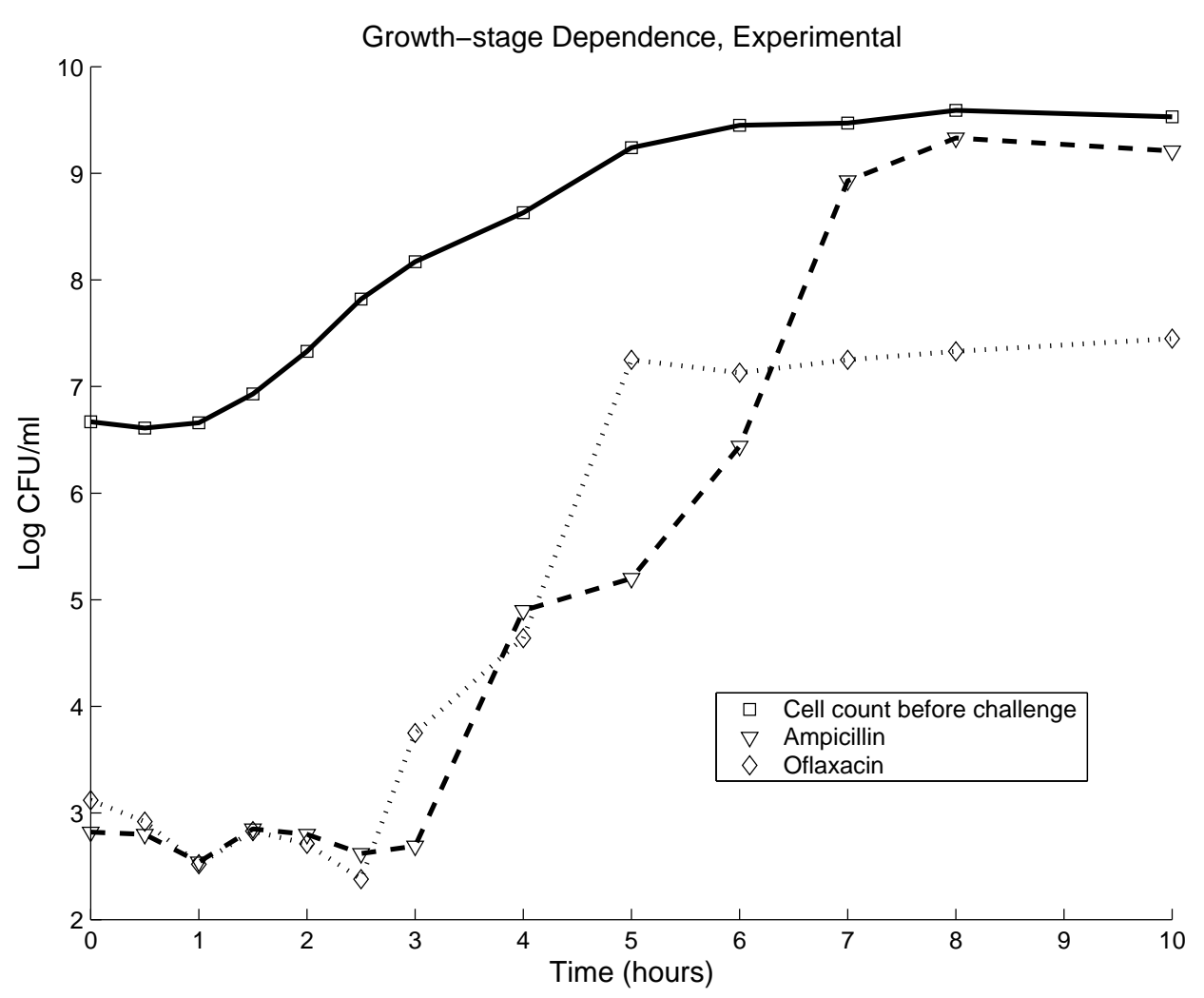

Figure 1: Growth-stage dependence of persister formation. The solid curve shows the time course of an unchallenged population of bacteria. At designated times samples were taken and enumerated after three hour exposure to to ampicillin (triangles) or oflaxacin (diamonds). The data for the broken lines are not time-courses but indicate the dependence of persister expression on the growth-stage. Data courtesy of K. Lewis.

bacteria were cultured in a shaker overnight. At designated times $1 \mathrm{ml}$ of cells were treated with antibiotics for three hours and the surviving bacteria were enumerated. The timedependent killing of exponential phase bacteria in the exponential phase was determined by incubating a culture and then exposing the cells to antibiotics. The population was then enumerated at specified times. The ratio between the surviving population and the initial population gave a measure of the effectiveness of the antibiotic. Survival curves show that within 0.5-1 hour more than $99 \%$ of the cells were killed. Continued exposure had no significant effect on the surviving population indicating the existence of 'persister cells'.

The investigators then show that the persisters cells are not mutants by re-suspending the bacteria and incubating them with aeration for 16-24 hours. The survival curves are the same as that of the initial exposure. Next the growth-state dependence of persisters was determined by challenging samples of a growing culture at designated times. The results indicate that persister formation was constant during the lag and early exponential phases. During the mid-exponential phase the number of persisters increased abruptly. Results from these experiments are shown in Figure 1.

In both studies antibiotics with different targets are used, a $\beta$-lactam and a fluoroquinolone. This is important, because fluoroquinolones are known to be effective against 
non-growing cells. This indicates that the resistance mechanism is more complicated than purely physiological resistance.

In the following sections we describe a mathematical model of the dynamics of the susceptible and persister bacterial cells with one growth limiting substrate. In the absence of antibiotic, the susceptible bacteria consume substrate and reproduce. When antibiotic is added, a fraction of the the susceptible cells are killed while another fraction convert to persister cells. Persister cells are not killed by the antibiotic nor do they grow. Instead, if there is no antibiotic, persister cells revert to susceptible cells at a fixed rate.

\section{Model Assumptions and Description}

We denote the bacterial phenotypes as $B_{s}$ and $B_{p}$ for susceptible and persister density, respectively. We assume that there is one growth-limiting substrate, $S$, and one antibiotic, $A$. The population of susceptible bacteria changes due to growth, death due to antibiotic action, loss due to transition to persister cells and gain as the persistent cells revert back to susceptible cells. Thus, the equation governing the dynamics of the susceptible population is given qualitatively as,

$$
\begin{aligned}
\frac{d B_{s}}{d t}= & \underbrace{g\left(B_{s}, S\right)}_{\text {Growth }}-\underbrace{d\left(B_{s}, S, A\right)}_{\text {Disinfection }} \\
& -\underbrace{l\left(B_{s}, S, A\right)}_{\text {Loss }}+\underbrace{r\left(B_{p}, S, A\right)}_{\text {Reversion }} .
\end{aligned}
$$

Growth is described by Monod kinetics with maximum specific growth rate, Monod coefficient and yield denoted $\mu_{\max }, K_{s}$ and $Y$, respectively. Thus the growth term is

$$
g\left(B_{s}, S\right)=\frac{\mu_{\max }}{Y} \frac{S}{K_{s}+S} B_{s}
$$

It has been shown experimentally that inactive or slowly growing bacteria are more tolerant to antibiotic exposure $[5,15]$. This observation has led to several models of disinfection which explicitly address this mechanism by linking the disinfection rate to the growth rate of the bacteria $[23,6]$. These studies indicate that physiological properties of bacteria play an important role in conferring tolerance.

Moreover, disinfection depends on the type of antibiotic used since fluoroquinolones are known to be partially effective in killing non-growing bacteria [21]. Therefore, if the antibiotic is a beta-lactam then the disinfection rate is assumed to be proportional to the growth rate. If the antibiotic is a fluoroquinolone we allow for disinfection in the absence of growth, although at a reduced rate. We model the disinfection term in Equation 1 as,

$$
d\left(B_{s}, S, A\right)=k_{d}(A, t) \mu_{\max } \frac{S+\alpha}{K_{s}+S} B_{s}
$$


where $\alpha$ is zero for beta-lactam and non-zero for fluoroquinolone. The function $k_{d}(A, t)$ depends on the antibiotic concentration. In particular, $k_{d}=0$ if $A=0$ and is nonzero otherwise. Since the dose strategies are time dependent, the disinfection rates also depend on time.

Although the mechanism that induces persister formation is not known, it is linked to the growth stage of the bacteria. One possible mechanism that could account for the abrupt change in the rate of persister formation is the presence of auto-regulatory signal. It is well known that expression of many genes associated with biofilm formation are regulated by such signals and can cause changes in the behavior of the bacteria[9]. Here we assume that the loss of susceptible cells to the persister population occurs at a rate that depends on both the growth rate and the antibiotic concentration. Mathematically we have,

$$
l\left(B_{s}, S, A\right)=k_{l}(A, t) \mu_{\max } \frac{S}{K_{s}+S} B_{s} .
$$

This incorporates the dependence of the persister formation rate on the growth-stage. In particular, when the population is in the stationary phase there is essentially no persister formation. During the exponential growth stage there is a relatively high rate of persister formation. Since the transition from susceptible to persister is assumed to be caused by exposure to the antibiotic, $k_{l}$ is also time dependent.

We assume that persister cells only revert to susceptible cells if there is no applied antibiotic. This assumption is also motivated by the experiments in [21] where susceptibility is recovered after several hours of incubation without antibiotic present while there is essentially no change in the population for continuous exposure. Mathematically we have $r\left(B_{p}, S, A\right)=k_{g}(A, t) B_{p}$, where $k_{g}$ is zero if there is antibiotic present and non-zero otherwise.

Putting these together gives the equation governing the dynamics of the susceptible population as

$$
\begin{aligned}
\frac{d B_{s}}{d t}= & \frac{\mu_{\max }}{Y} \frac{S}{K_{s}+S} B_{s}-k_{d}(A, t) \mu_{\max } \frac{S+\alpha}{K_{s}+S} B_{s} \\
& -k_{l}(A, t) \mu_{\max } \frac{S}{K_{s}+S} B_{s}+k_{g}(A, t) B_{p} .
\end{aligned}
$$

Persister cells are not killed by the antibiotic, instead the population changes as cells convert to and from susceptible cells,

$$
\frac{d B_{p}}{d t}=k_{l}(A, t) \mu_{\max } \frac{S}{K_{s}+S} B_{s}-k_{g}(A, t) B_{p}
$$

We assume that substrate is being consumed only by the susceptible population so the equation governing the substrate concentration is,

$$
\frac{d S}{d t}=-\mu_{\max } \frac{S}{K_{s}+S} B_{s}
$$

Equations 2 - 4 describe the dynamics for suspended populations of susceptible and persisting bacteria and substrate. In the next section, we describe simulations which yield 


\begin{tabular}{l|l|l|l|l}
\hline & & & \\
Parameter & Symbol & Units & Value & Source \\
\hline Maximum Specific Growth Rate & $\mu_{s}$ & $\mathrm{~h}^{-1}$ & 0.417 & {$[23]$} \\
Yield Coefficient & $Y$ & & 0.2 & {$[23]$} \\
Monod Coefficient & $K_{s}$ & $\mathrm{mg} \mathrm{l}^{-1}$ & 0.2 & {$[23]$} \\
Maximum Disinfection Rate & $k_{d}$ & $\mathrm{~h}^{-1}$ & 40 & Estimated \\
Non-growing Disinfection & $\alpha$ & $\mathrm{mg} \mathrm{l}^{-1}$ & 0.07 & Estimated \\
Rate of Loss & $k_{l}$ & $\mathrm{~h}^{-1}$ & 0.001 & Estimated \\
Rate of Gain & $k_{g}$ & $\mathrm{~h}^{-1}$ & 0.05 & Estimated \\
\hline
\end{tabular}

Table I: Parameters used in the simulations.

results comparable to experimental results shown in [17]. Then we show results from a simulated dosing protocol entailing application of a constant concentration of antibiotic for a specified length of time, withdrawing the antibiotic and allowing the population to regrow. The results depend dramatically on the length of dose/withdrawal times. In particular, for short withdrawal times there is a persister population generated which is eliminated extremely slowly. If the treatment is terminated before the persister population is cleared, there is rapid regrowth of the bacterial population. For long withdrawal times the susceptible population is only transiently eliminated. The persister cells, generated from the dosing step, are a source for the susceptible population which reproduce. There are intermediate withdrawal times where neither of these cases occurs.

Following this section we introduce a simplified model which is amenable to analytic treatment. The reduced model still retains the fundamental behavior of successful treatment for intermediate dose/withdrawal times.

\subsection{Parameters}

Table I lists the parameters and values. We have used typical values for maximum growth rate, yield and Monod coefficient. The four parameters which depend on the antimicrobial agent, $k_{d}, \alpha, k_{l}$ and $k_{g}$ were estimated. We assume that the rates are linearly proportional to $A$ and we determined the constants of proportionality.

The parameters regarding disinfection rate and the rate of transformation from susceptible to persister cells were chosen to fit the time-dependent kill curve for constant exposure found in [17] (see Figure 2). In the experiment antibiotic was applied continuously so $k_{d}$ and $k_{l}$ are constant in time. Since we assume that there is no reversion from persistent to susceptible whenever there is antibiotic applied the reversion rate, $k_{g}$, is zero. The values of $k_{d}$ and $k_{l}$ are chosen to match the time-scale for the plateau region and also the effectiveness of each antibiotic. Comparing the predicted survival curves with the data indicate that our model is capturing both the gross qualitative trends as well as the correct scales.

Once the parameters $k_{d}$ and $k_{l}$ have been fixed, we simulate the growth-stage dependence of persister formation. In the experiments, bacteria were disinfected as a suspension. Periodic samples were taken from the reactor and exposed to antibiotic and then enumerated. This generates two curves, an untreated growth curve and a treated growth curve. It should be 


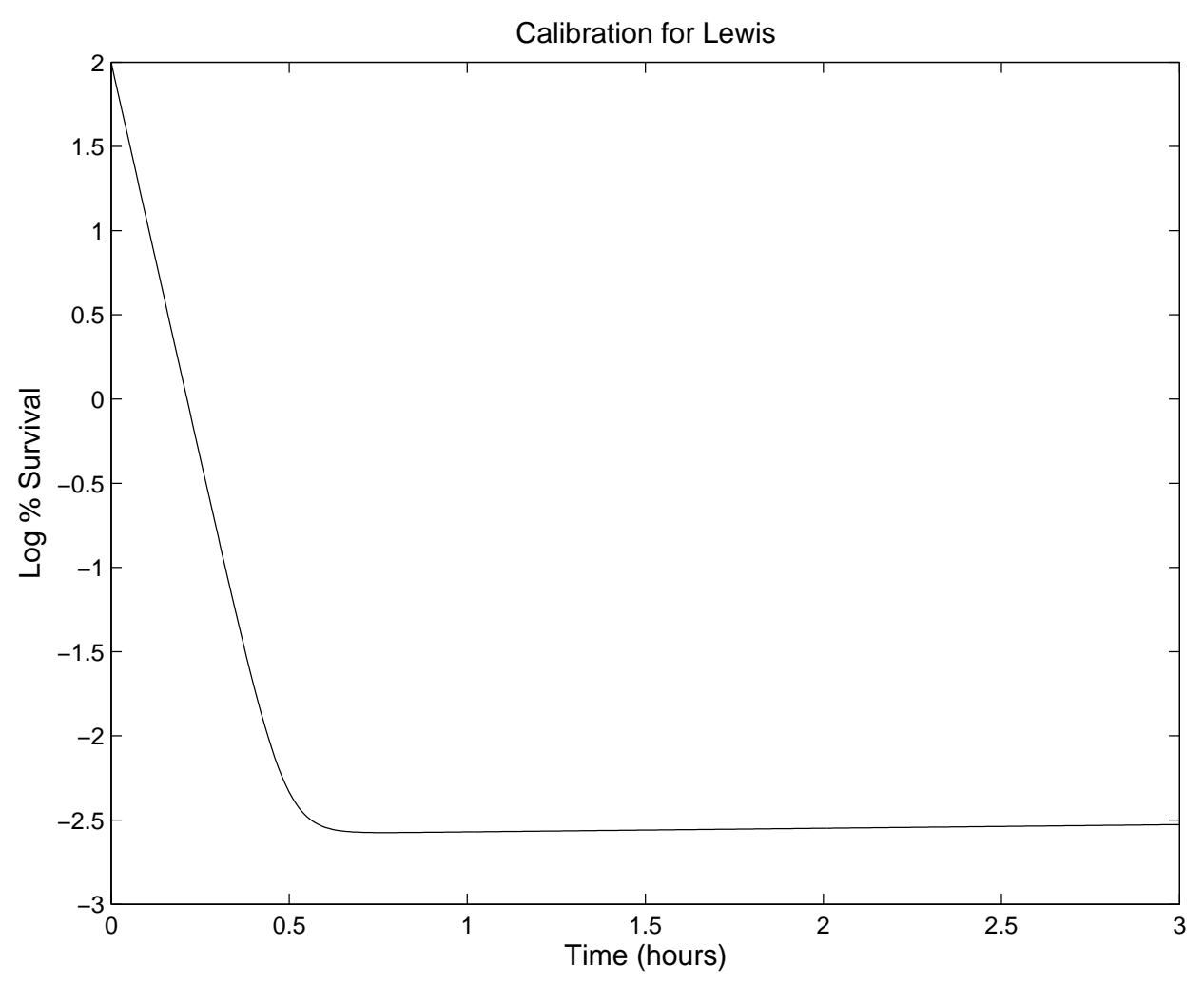

Figure 2: Time-dependent killing curve for cells treated continuously with antibiotic. Here the disinfection parameters are constant in time, since there is always antibiotic present. This qualitatively agrees with data from [17] and shows the distinct bi-phasic nature, typical for antibiotic treatment. We are primarily concerned with the time scale associated with clearance and our parameters have been chosen so that the plateau region begins within the first $\frac{1}{2}$ hour. The parameters are listed in Table I. 
noted that the treated growth curve is not a time-series, but rather a representation of the estimated persister population for various stages of growth. In our simulations, we began with initial conditions corresponding to $1 \times 10^{7}$ susceptible cells, zero persister cells and substrate concentration of twice $K_{s}$.

In these simulations the antibiotic is either never applied (to generate an untreated growth curve) or applied continuously to bacteria at particular growth stages. Thus the parameters are not time dependent, rather there are separate experiments being performed, one with no antibiotic being applied and those where antibiotic is being applied continuously to bacteria in fixed stages of growth. The untreated growth curve is determined by solving Equations 2 - 4 using the package ODE45 in MATLAB with $k_{d}, k_{g}$ and $k_{l}$ zero. Once the untreated simulation is completed, we use values of $B_{s}, B_{p}$ and $S$ at specified times, and simulate the effect of constant exposure to antibiotic for three hours. Our simulation results for both $\beta$-lactam and fluoroquinolone antibiotics are shown in Figures 3 and 4.

Finally, we assume that persister cells lose their persister phenotype after several generations which yields an estimate for $k_{g}$. The results shown below do not depend qualitatively on the estimate for $k_{g}$, rather it is only necessary that persisters are capable of reverting back to susceptible.

\subsection{Simulated Dosing Experiment - ODE}

In this section we describe results from a simulated dosing experiment motivated by comments in [21]. The author describes a possible treatment that requires applying an antibiotic for a period of time, killing all susceptible bacteria while generating small population of persister cells. Withdrawing the antibiotic allows the persister cells to grow and revert back to susceptible cells. At this point a second application will remove virtually all of the bacteria.

We begin our simulations with a population of susceptible cells which are exposed to nutrient and an antibiotic for a fixed length of time, denoted $T_{d}$. The nutrient is initially twice $K_{s}$. The susceptible cells are quickly eliminated and a small population of persister cells is produced. The antibiotic is removed allowing the cells to grow for a fixed length of time, $T_{w}$. Persister cells revert to susceptible cells, which then consume nutrient and reproduce. This completes one dose/withdrawal period. After one dose/withdrawal period, fresh nutrient is added and the cycle is repeated. Results from numerical experiments, with a dose period of 10 hours and varying withdrawal period are shown in Figures 5 - 7 . If the bacterial population is not eliminated within approximately 30 days the treatment is deemed ineffective and the simulation is stopped.

For short withdrawal periods the treatment fails to eliminate the persister population within the alloted time period (see Figure 5). If the treatment is discontinued, the population quickly regrows. For large withdrawal periods the susceptible population is not eliminated. Instead, the susceptible population responds to the treatment quickly while generating a persister population. The persisters are a source of susceptible cells once the antibiotic is withdrawn. This results in an ineffective treatment (see Figure 6). There are intermediate dose/withdrawal pairs for which the treatment is effective in clearing both the susceptible and persister population (see Figure 7). This indicates that periodic dosing may be effective,

but the dosing regiment must be specific to the bacterial kinetics. The withdrawal period must be long enough to allow the persister cells to revert to susceptible cells but not long 


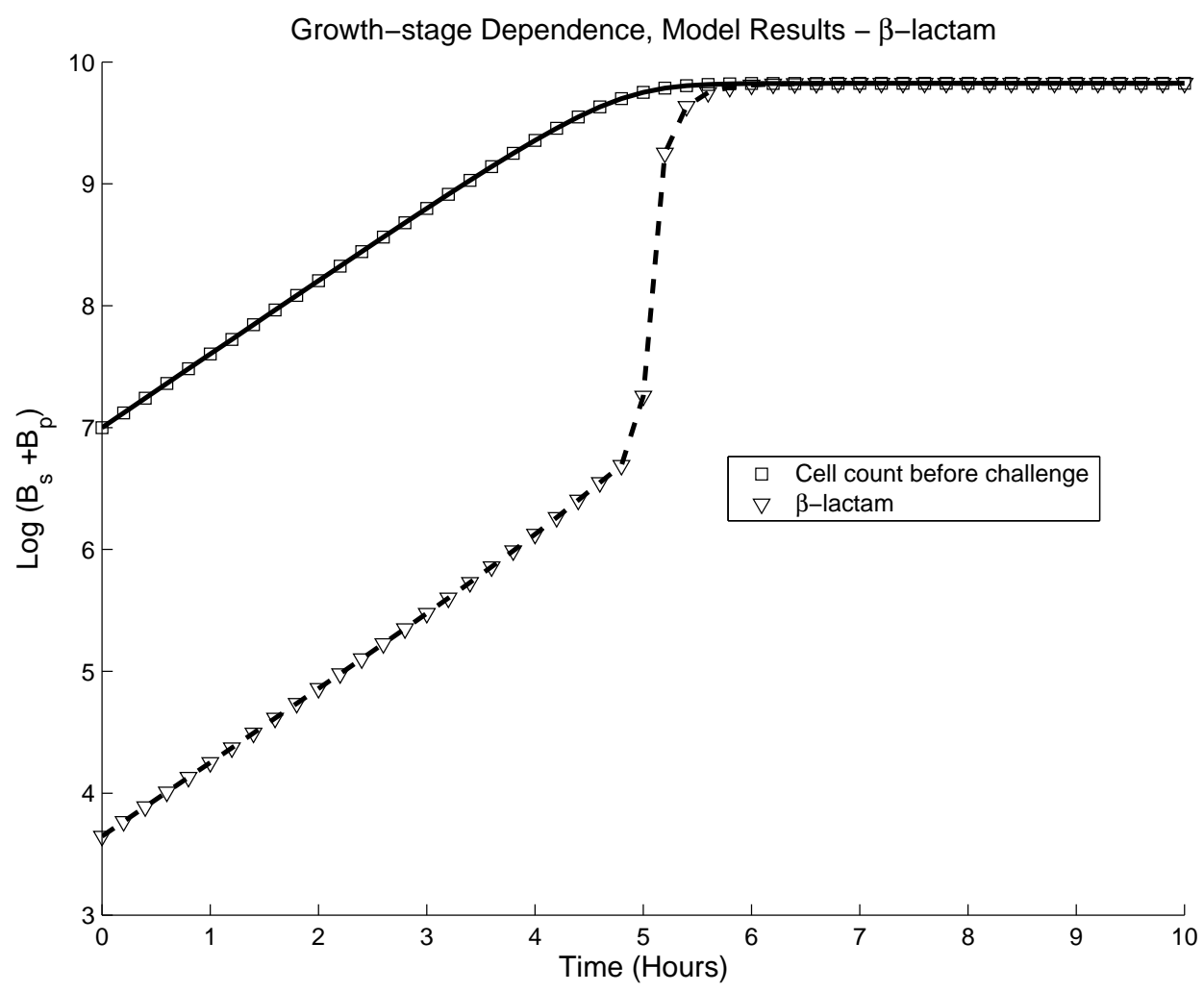

Figure 3: Growth-stage dependence of persister formation for bacteria challenged with a $\beta$-lactam (growth-rate dependent) antibiotic. The squares represent the cell count before challenge and the triangles are the cell count after the challenge. The solid curve corresponds to the untreated population, so the disinfection parameters $k_{d}$ and $k_{l}$ are zero. The dashed curve is generated by taking bacteria at indicated and exposing them to a constant level of antibiotic ( $k_{d}$ and $k_{l}$ nonzero) for three hour and interpolating the data. We see an abrupt increase in the number of surviving bacteria at approximately five hours indicating an increase in the number of persister type bacteria. Our results agree well with the data in [17]. We also see that as the growth-rate goes to zero, because of nutrient depletion, the entire population becomes tolerant. 


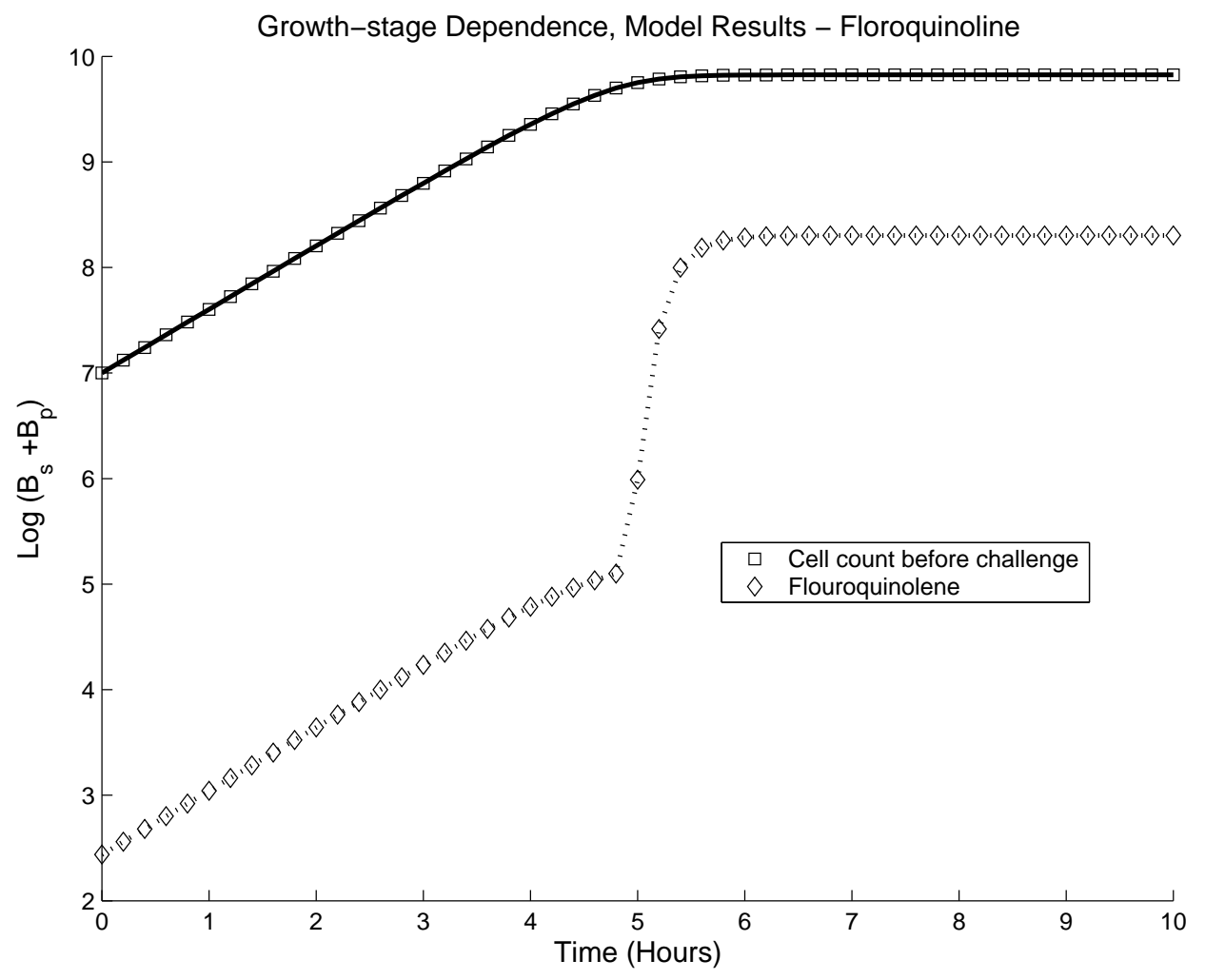

Figure 4: Growth-stage dependence of persister formation for bacteria challenged with a fluoroquinolone (non-growth-rate dependent) antibiotic. The squares represent the cell count before challenge and the diamonds are the cell count after the challenge. The solid curve corresponds to the untreated population, so the disinfection parameters $k_{d}$ and $k_{l}$ are zero. The dashed curve is generated by taking bacteria at indicated and exposing them to a constant level of antibiotic ( $k_{d}$ and $k_{l}$ nonzero) for three hour and interpolating the data. Again, we see an abrupt increase in the number of surviving bacteria at approximately five hours. Our results agree well with the data in [17]. Because the antibiotic is effective against non-growing cells, we do not see saturation to the untreated cell count as in Figure (3). Instead, the cell count saturates to a lower level. 

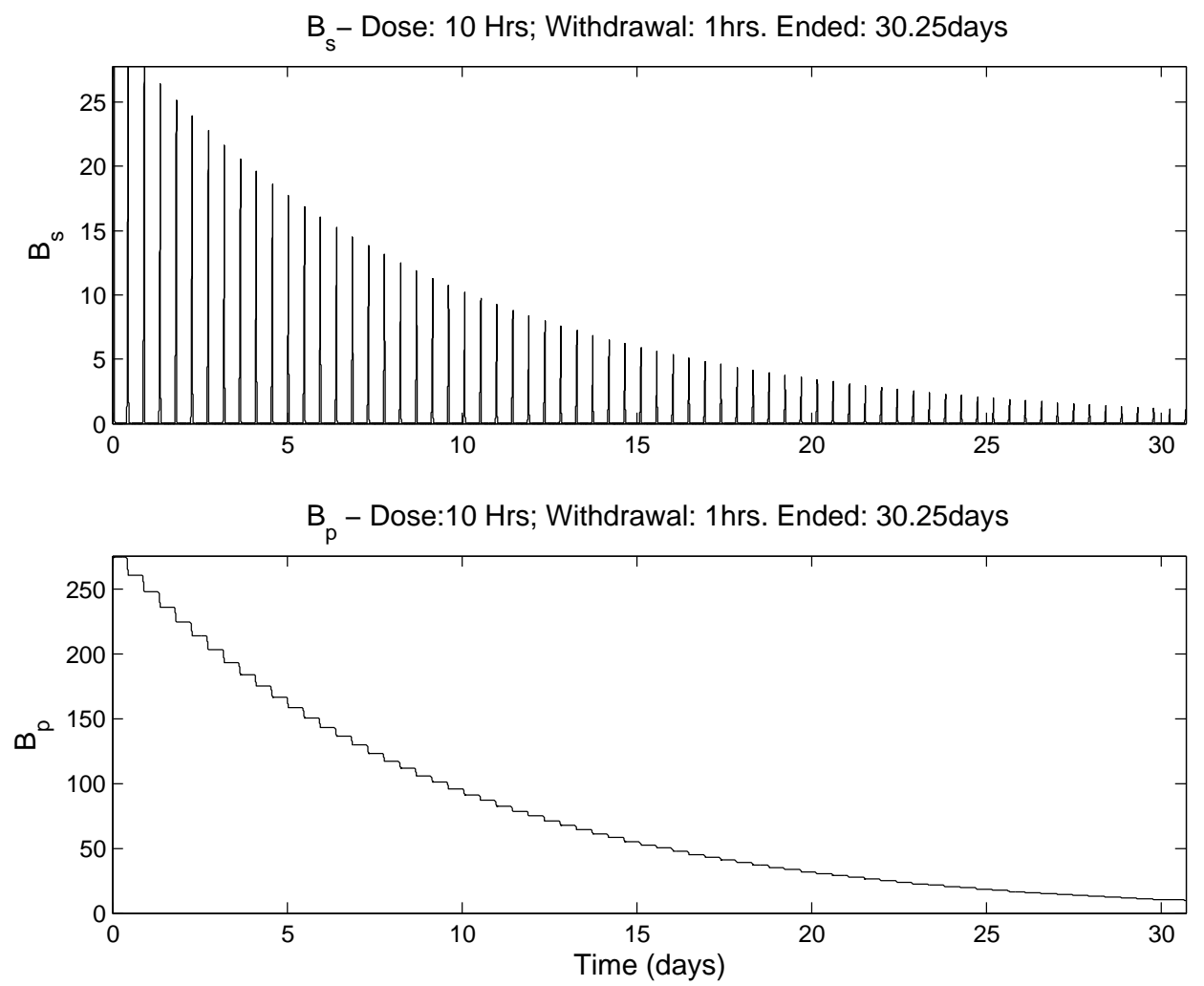

Figure 5: Periodic dosing experiment with withdrawal period of one hour. We see that, while the susceptible cells are killed, the persister population is being killed very slowly. This treatment was not successful. 

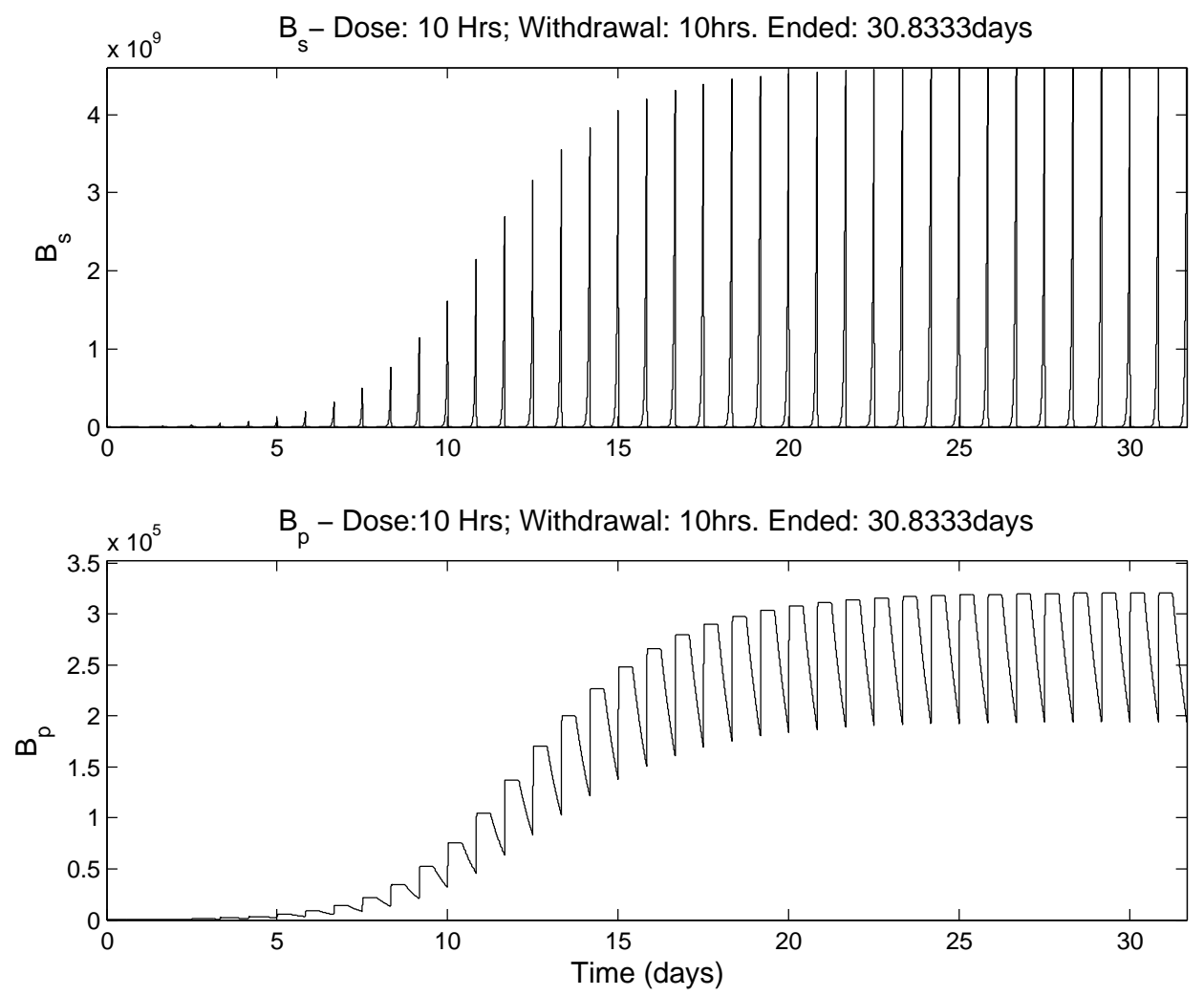

Figure 6: Periodic dosing experiment with withdrawal period of ten hours. We see that neither the susceptible nor the persister cells are eliminated. This treatment was not successful. 

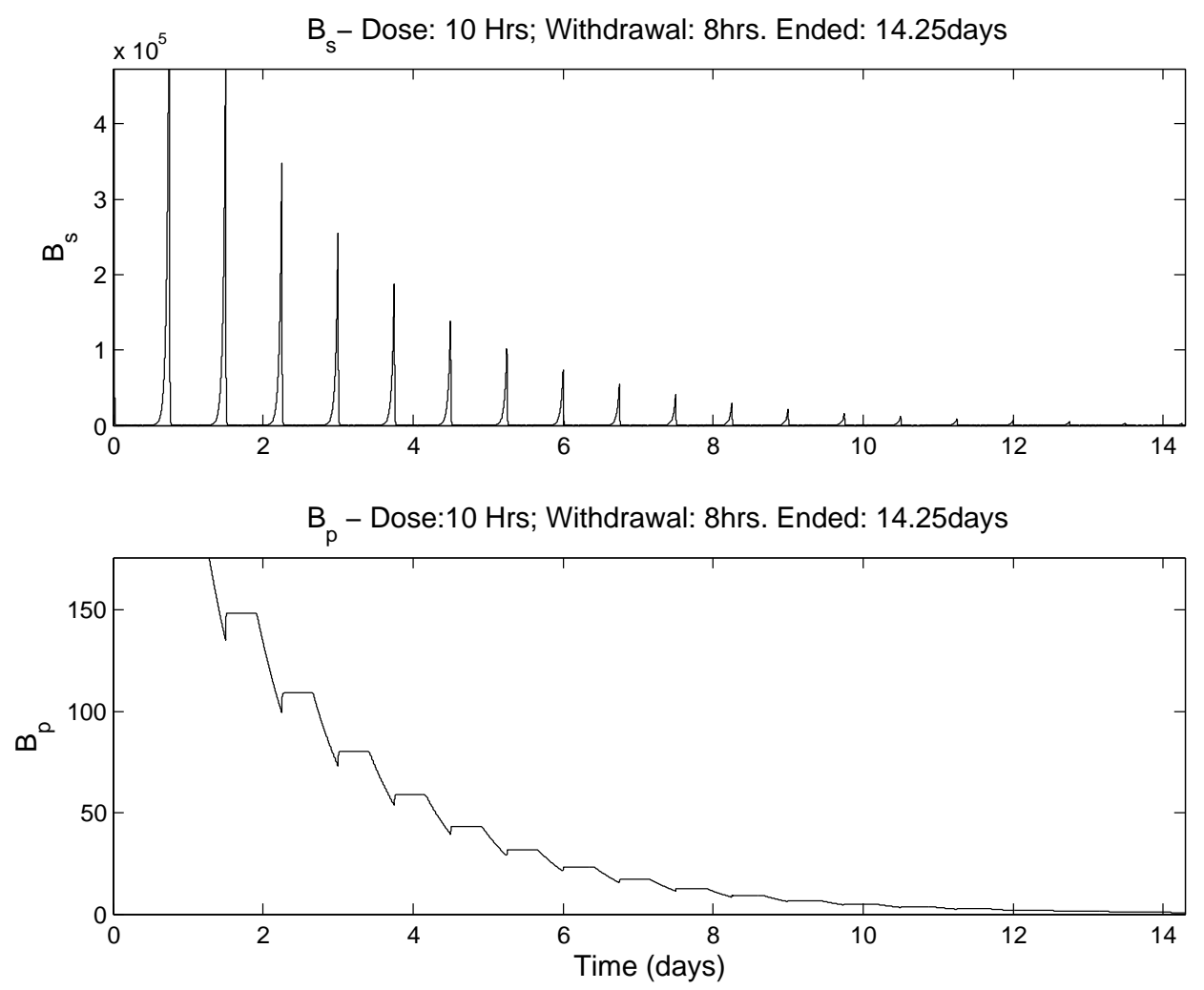

Figure 7: Periodic dosing experiment with withdrawal period of eight hours. We see that both the susceptible and persister cells are killed indicating a successful treatment. 

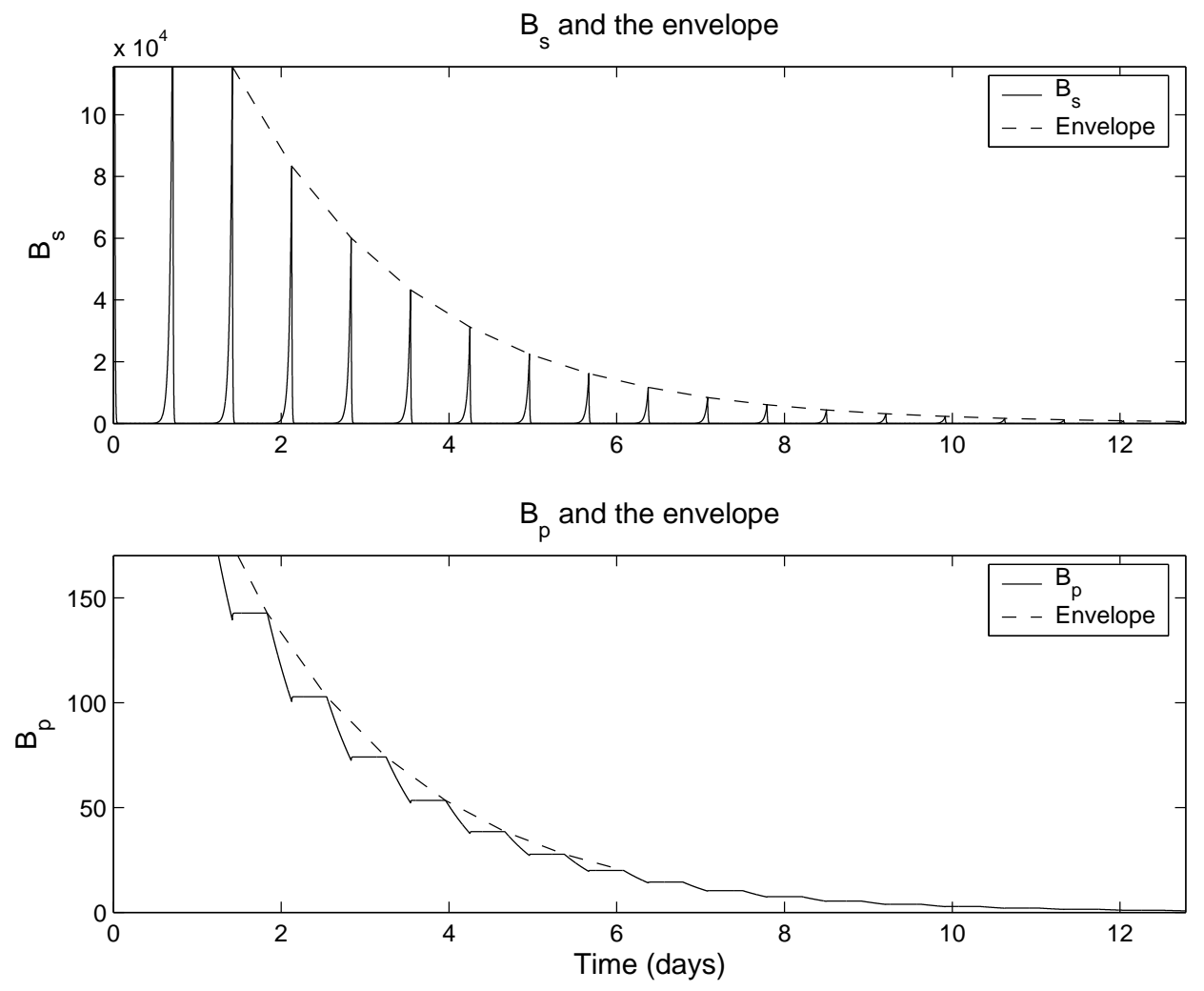

Figure 8: Survival curves for susceptible and persister cells along with the envelopes of the curves. The envelopes are given by the maximum of the populations during the dosing cycle. This gives an overall view of the effect of the dosing strategy.

enough for the susceptible cells to reach the exponential growth stage.

To find the optimal withdrawal length, we measure the change in population as a function of this length. The envelope of the survival curves is the maximum of the respective populations during each cycle (see Figure 8). The slope of the envelope on a logarithmic scale gives the exponential rate of change in the population. For successful treatments, the maximum rate is negative. In Figure 9, we show the maximum slope of the logarithm of the envelope as a function of the withdrawal time. Minimizing this curve gives the optimal strategy for the parameters given in Table $\mathrm{I}$ and $T_{d}=10$ hours. In this care the optimal value for $T_{w}$ is approximately 7.5 hours.

Results from the simulations predict that there is an optimal dosing strategy. However, because the differential equations are nonlinear and non-autonomous it is difficult to proceed with any analytic treatment. In the following section, we simplify the model by assuming that the nutrient level is constant. This reduces the model from a system of three coupled nonlinear, non-autonomous equations to a system of two linear, non-autonomous equations. These equations can be solved analytically and the dynamics of the populations of susceptible and persistent bacteria reduce to a two-dimensional map. The success of the dosing strategy is determined by the eigenvalues of the map. 


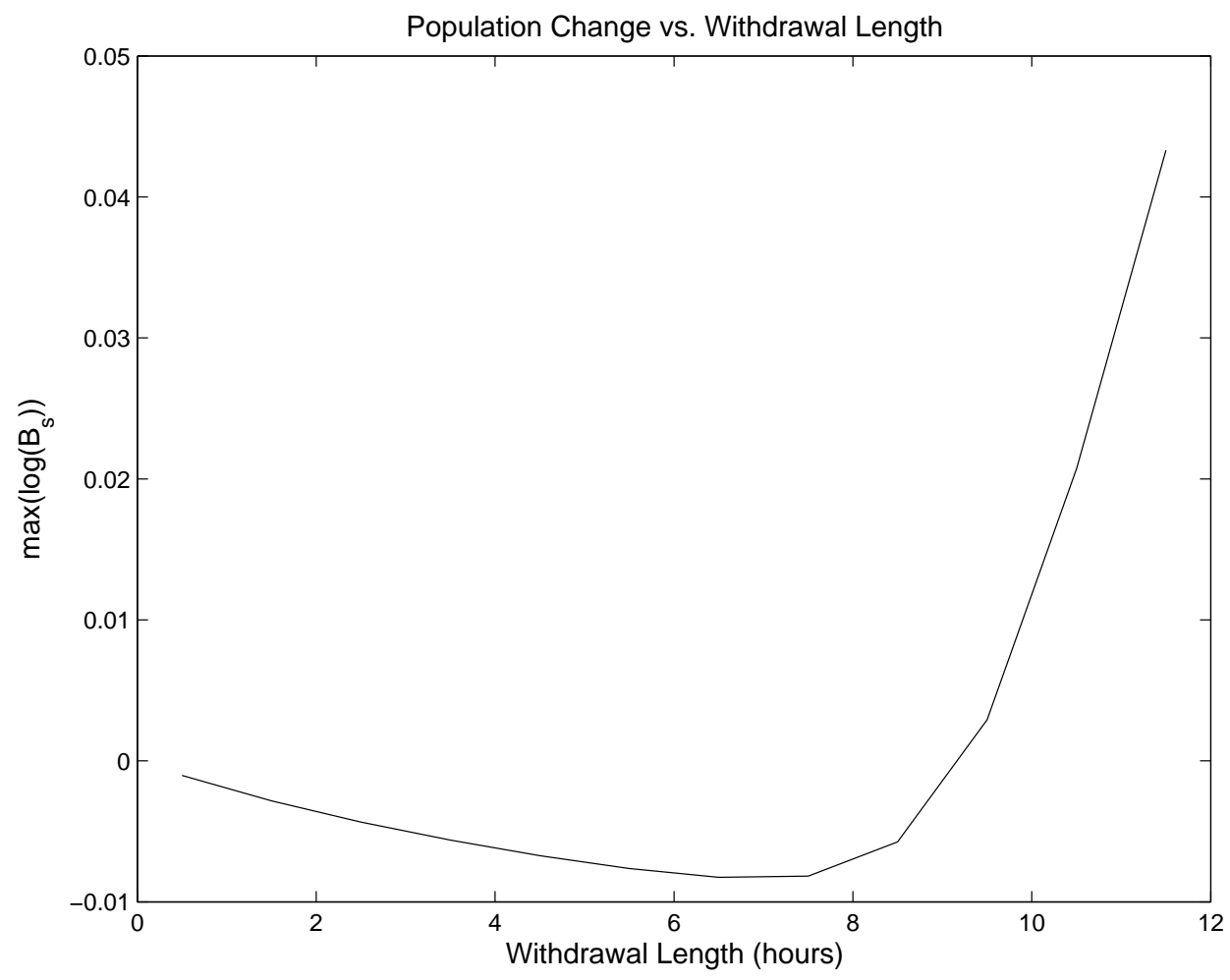

Figure 9: Maximum rate of change for the population of cells as a function of the withdrawal length. The maximum rate of change of envelope of the population is calculated for various withdrawal lengths. We see that for withdrawal time shorter than approximately 9 hours, the treatment is effective. For withdrawal periods longer than this, the overall population actually increases. The minimum of this curve gives the optimal withdrawal time as approximately 7.5 . 


\section{Simplified Model}

Assuming that the nutrient concentration is constant in time gives,

$$
\begin{aligned}
\frac{d B_{s}}{d t}= & \hat{k}_{s} B_{s}-\hat{k}_{d}(A, t) B_{s} \\
& -\hat{k}_{l}(A, t) B_{s}+\hat{k}_{g}(A, t) B_{p} \\
\frac{d B_{p}}{d t}= & \hat{k}_{l}(A, t) B_{s}-\hat{k}_{g}(A, t) B_{p} .
\end{aligned}
$$

We further assume that the antibiotic level switches instantaneously from application to withdrawal. Under these assumptions, $\hat{k}_{s}$ is a positive constant while $\hat{k}_{d}$ and $\hat{k}_{l}$ are zero if there is no antibiotic and non-zero otherwise. The rate of reversion from persister to susceptible, $\hat{k}_{g}$, is non-zero only if the antibiotic is not applied. The solution to Equations 5 and 6 with initial conditions $B_{s}(0)$ and $B_{p}(0)$ are

$$
B_{s}(t)= \begin{cases}B_{s}(0) e^{\left(\hat{k}_{r}-\hat{k}_{d}-\hat{k}_{l}\right) t} & A \geq 0 \\ \frac{\left(B_{s}(0)+B_{p}(0)\right) \hat{k}_{g}+B_{s}(0) \hat{k}_{r}}{\hat{k}_{g}+\hat{k}_{r}} e^{\hat{k}_{r} t}-\frac{B_{p}(0) \hat{k}_{g}}{\hat{k}_{g}+\hat{k}_{r}} e^{-\hat{k}_{g} t} & A=0\end{cases}
$$

and

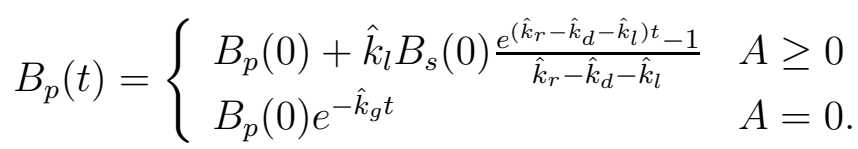

We begin with an initial population of bacteria which are all susceptible (i.e. $B_{s}(0)=$ $B_{s}, B_{p}(0)=0$ ) and apply an antibiotic for a length of time $T_{d}$. The population of each phenotype is,

$$
\begin{aligned}
& B_{s}\left(T_{d}\right)=B_{s}(0) e^{\left(\hat{k}_{r}-\hat{k}_{d}-\hat{k}_{l}\right) T_{d}} \\
& B_{p}\left(T_{d}\right)=\hat{k}_{l} B_{s}(0) \frac{e^{\left(\hat{k}_{r}-\hat{k}_{d}-\hat{k}_{l}\right) T_{d}}-1}{\hat{k}_{r}-\hat{k}_{d}-\hat{k}_{l}} .
\end{aligned}
$$

Using these values as initial conditions for the dynamics with $A=0$ for a length of time $T_{w}$ we obtain the new populations,

$$
\begin{aligned}
& B_{s}\left(T_{w}\right)=\frac{\left(B_{s}\left(T_{d}\right)+B_{p}\left(T_{d}\right)\right) \hat{k}_{g}+B_{s}\left(T_{d}\right) \hat{k}_{r}}{\hat{k}_{g}+\hat{k}_{r}} e^{\hat{k}_{r} T_{w}}-\frac{B_{p}\left(T_{d}\right) \hat{k}_{g}}{\hat{k}_{g}+\hat{k}_{r}} e^{-\hat{k}_{g} T_{w}} \\
& B_{p}\left(T_{w}\right)=B_{p}\left(T_{d}\right) e^{-\hat{k}_{g} T_{w}}
\end{aligned}
$$

We define $M$ as the map taking $\left(B_{s}(0), B_{p}(0)\right)$ to $\left(B_{s}\left(T_{w}\right), B_{p}\left(T_{w}\right)\right)$ which can be written as a matrix with entries

$$
\begin{aligned}
M_{1,1} & =\frac{\left(\hat{k}_{r}^{2}+\hat{k}_{g} \hat{k}_{r}-\hat{k}_{r} \hat{k}_{d}-\hat{k}_{g} \hat{k}_{l}-\hat{k}_{g} \hat{k}_{d}\right) e^{\hat{k}_{r} T_{w}+\left(\hat{k}_{r}-\hat{k}_{d}-\hat{k}_{l}\right) T_{d}}-\hat{k}_{g} \hat{k}_{l} e^{-\hat{k}_{1} T_{w}}}{\left(\hat{k}_{g}+\hat{k}_{r}\right)\left(\hat{k}_{r}-\hat{k}_{d}-\hat{k}_{l}\right)} \\
M_{1,2} & =\frac{\hat{k}_{g}}{\hat{k}_{g}+\hat{k}_{r}}\left(e^{\hat{k}_{r} T_{w}}-e^{-\hat{k}_{g} T_{w}}\right) \\
M_{2,1} & =\frac{\hat{k}_{l}}{\hat{k}_{r}-\hat{k}_{d}-\hat{k}_{l}}\left(e^{\left(\hat{k}_{r}-\hat{k}_{d}-\hat{k}_{l}\right) T_{d}}-1\right) e^{-\hat{k}_{g} T_{w}} \\
M_{2,2} & =e^{-\hat{k}_{g} T_{w}} .
\end{aligned}
$$




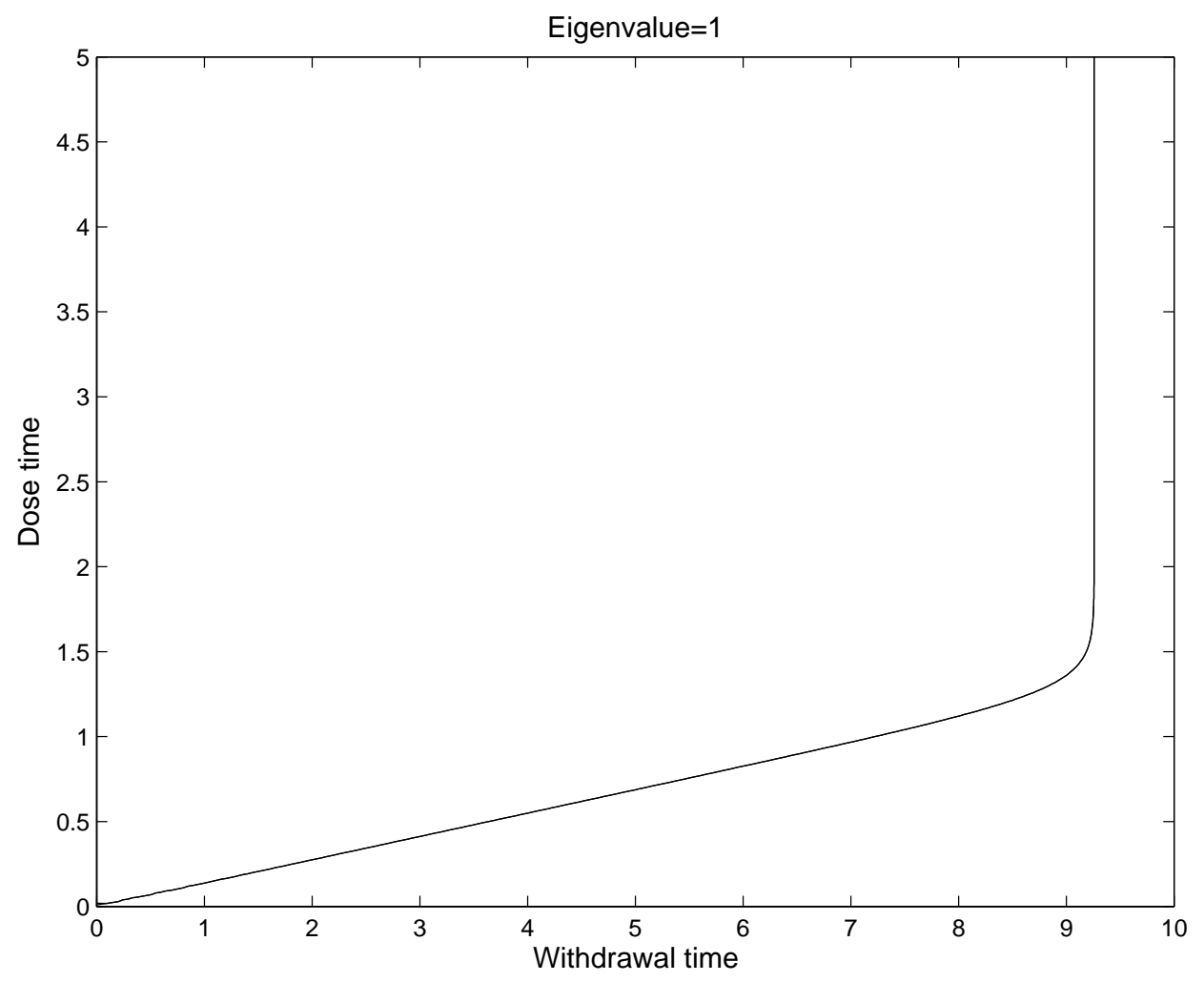

Figure 10: The curve in the $T_{d}, T_{w}$-plane for which the eigenvalue is equal to one. Above this curve, the eigenvalue is less than one, while below this curve the eigenvalue is greater than one.

The success or failure of the dosing strategy in this simplified situation is determined by the eigenvalues of the map $M$. If the magnitude of each eigenvalues is less than one, the repeated dose/withdrawal will eventually eradicate both populations. Otherwise there will be growth of one or both of the phenotypes, indicating an unsuccessful outcome.

We find that one of the eigenvalues is less than one for all nonzero dose/withdrawal times. The curve in the $\left(T_{d}, T_{w}\right)$-axis for which the second eigenvalue is equal to one is shown in Figure 10. Above this curve the eigenvalue is less than one, while below this curve the eigenvalue is greater than one. We also show the value of the eigenvalue for fixed dose time and varying withdrawal times in Figure 11. This curve agrees qualitatively with the simulations shown in Section 3.2. In particular, we see that for short withdrawal times we have successful treatment. The rate at which the populations decrease to zero for varying $T_{w}$ has a unique minimum. Both the longest successful withdrawal time and the optimal withdrawal time are similar to those obtained in Section 3.2.

We also find that for withdrawal time larger than approximately 9.3 there is no successful treatment. Although the results are shown only for $T_{d}$ less than 5 hours, this conclusion is true for much larger values (i.e. $T_{d}>40$ hours). The withdrawal time along with unbounded growth produces too many bacteria for the treatment to be effective. 


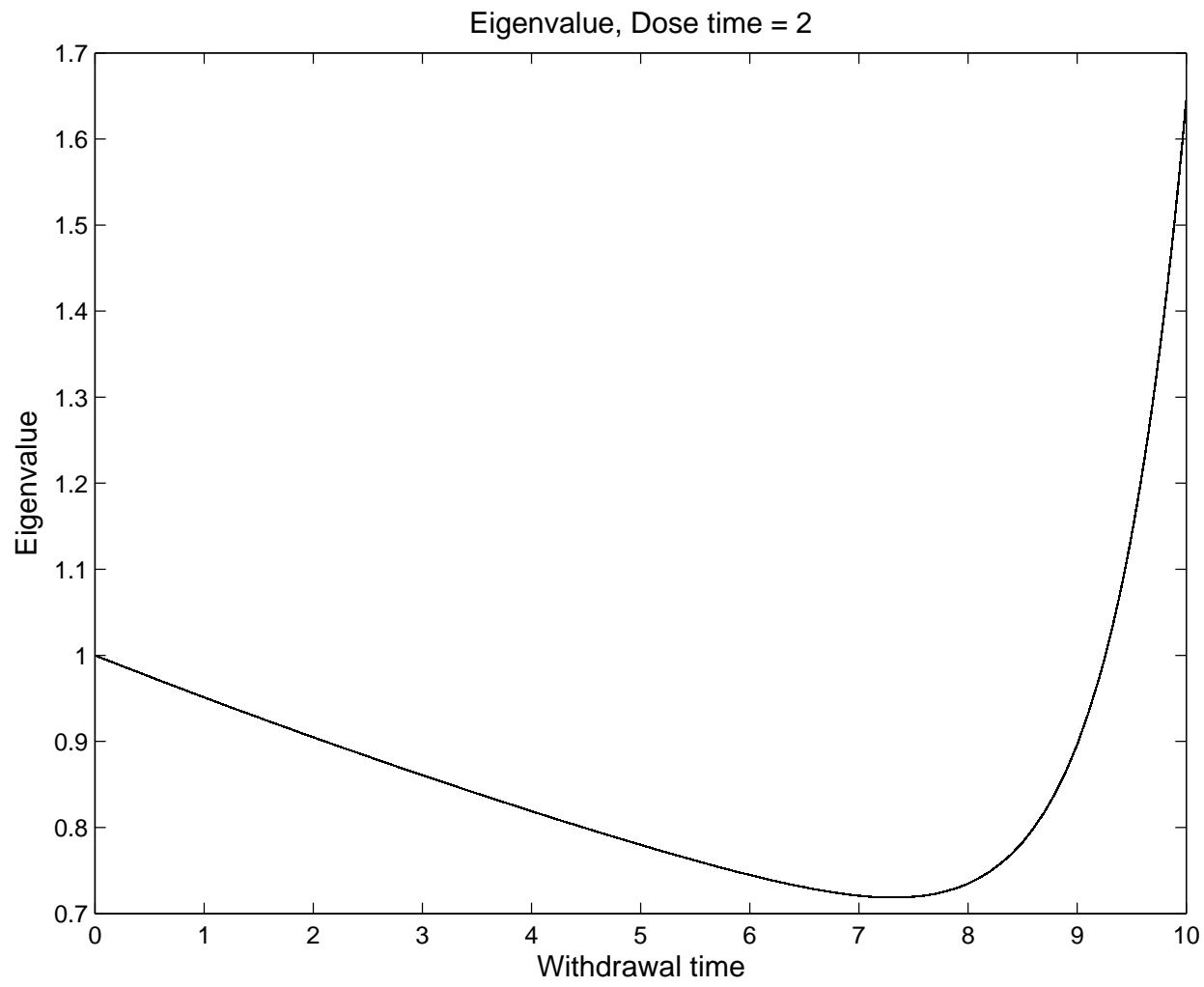

Figure 11: The largest eigenvalue for fixed $T_{d}=2$ as a function of the withdrawal time. For withdrawal times less than approximately nine hours, the eigenvalue is less than one, indicating a successful treatment. The minimum value occurs at approximately 7.5 hours, which agrees well with results from Section 3.2. 


\section{Discussion}

We have presented a mathematical model of bacterial tolerance based experimentally observed 'persister cells'. These cells are a dormant phenotype that is expressed at a rate that depends on the population growth rate and the antibiotic concentration. Parameters of the model have been chosen to yield results comparable to experiments.

We use the model to test the hypothesis that alternating dose/withdrawal of the antibiotic results in elimination of all bacteria. The optimal strategy is also determined. Results of this analysis do not depend on the mechanism which induces the expression of the persistent phenotype. It is only necessary that the formation of persisters is related to the growth stage. This does not address the more difficult question concerning the regulation of persister expression. It seems reasonable to suppose that rather than linking the rate of formation of persisters to the growth rate there is a quorum sensing signal. Thus there may be a switch analogous to that which governs the expression of the biofilm phenotype.

Because we assume that the rate of persister formation is proportional to the growth rate, we find that persister cells are formed throughout the growth-cycle. This is not reflected in experimental results (Dr. Kim Lewis personal communication). Instead, it seems that there is no persister formation until the mid to late exponential phase. This may indicate that the expression of the persister phenotype is regulated by an auto-inductive signal

The specific mechanism governing the reversion from persister back to susceptible phenotype does not qualitatively alter the results of the analysis. As long as there is some reversion from persister back to susceptible the persister cells will act as a source for the regrowth of the bacterial population. This is a major problem for bacterial biofilm infections, since persister cells within the biofilm are also physically protected from the immune system by the surrounding polymer gel.

The dynamics of persister formation are clearly more complicated in a biofilm setting. In a recent paper [24], the authors consider a model of persister formation within a growing biofilm. Results from model simulations suggest that persisters can accumulate within the biofilm although the production of persisters near the surface of the biofilm is outpaced by reproduction of susceptible bacteria. If the rate of formation of persisters is instead related to the antibiotic and the growth rate the question is much more delicate since both the nutrient and the antibiotic must diffuse through the biofilm. The concentrations of antibiotic and nutrients depend on reactions within the biofilm which can alter the dynamics of persister formation.

In conclusion, we have shown that both persister and susceptible cells can be eliminated from a suspended population by an alternating dose/withdrawal strategy. It remains to be determined whether the same strategy will be effective in a biofilm setting where the transport of nutrient and antibiotic is more complicated. 


\section{References}

[1] D. Allison And P. GILBERT, Modification by surface association of antimicrobial susceptibility of bacterial populations, Journal of Industrial Microbiology, 15 (1995), pp. 311-317.

[2] M. Ashby, J. NeAle, AND I. CRITChley, Effect of antibiotics on non-growing planktonic cells and biofilms of Eschericha coli, Journal of Antimicrobial Chemotherapy, 33 (1994), pp. 443-452.

[3] N. Bagge, M. Hentzer, J. B. Anderson, O. Ciofu, M. Givskov, And N. HoIBy, Dynamics and spatial distribution of $\beta$-lactamase expression in Pseudomonas aeruginosa biofilms, Antimicrobial Agents and Chemotherapy, 48 (2004), pp. $1168-1174$.

[4] A. Brooun, J. Tomashek, And K. Lewis, A dose-response study of antibiotic resistance in Pseudomonas aeruginosa biofilm, Antimicrobial Agents and Chemotherapy, 44 (2000), pp. 640-646.

[5] M. Brown, D. Allison, And P. Gilbert, Resistance of bacterial biofilms to antibiotics: a growth-rate related effect?, J. Antimicrob. Chemother., 22 (1988), pp. 777-783.

[6] N. Cogan, R. Cortez, And L. Fauci, Modeling physiological resistance in bacterial biofilms, Bulletin of Mathematical Biology, 67 (2005), pp. 831-853. to appear.

[7] J. Costerton, P. S. Stewart, and E. P. Greenberg, Bacterial biofilms: A common cause of persistent infections, Science, 284 (1999), pp. 1318-1322.

[8] D. DAviEs, Understanding biofilm resistance to antibacterial agents, Nature Reviews Drug Discovery, 2 (2003), pp. 114-122.

[9] D. Davies, M. Parsek, J. Costerton, J. Pearson, B. Igleski, and E. GreenBERG, The involvement of cell-to-cell signals in the development of a bacterial biofilm, Science, 280 (1998).

[10] D. DeBeer, R. Srinivasan, And P. S. Stewart, Direct measurement of chlorine penetration into biofilms during disinfection, Applied Environmental Microbiology, 60 (1994), pp. 4339-4344.

[11] M. Desai, T. Buhler, P. Weller, And M. Brown, Increasing resistance of planktonic and biofilm cultures of Burkholderia cepeciax to ciproflaxacin and ceftazidime during exponential growth, Journal of Antimicrobial Chemotherapy, 42 (1998), pp. 153-160.

[12] M. G. Dodds, K. J. Grobe, and P. S. Stewart, Modeling biofilm antimicrobial resistance, Biotechnology and Bioengineering, 68 (2000), pp. 456-465.

[13] E. Drenkard And F. M. Ausubel, Pseudomonas biofilm formation and antibiotic resistance are linked to phenotypic variation, Nature, 416 (2002), pp. 740-743. 
[14] J. G. Elkins, D. J. Hassett, P. S. Stewart, H. P. Schweizer, and T. R. McDermott, Protective role of catalase in Pseudomonas aeruginosa biofilm resistance to hydrogen peroxide, Applied and Environmental Microbiology, 65 (1999), pp. 45944600 .

[15] P. Gilbert, T. Maira-Litran, A. J. McBain, A. H. Rickard, and F. W. WhYTE, The physiology and collective recalcitrance of microbial biofilm communities, Advances in Microbial Physiology, 46 (2002), pp. 202-256.

[16] B. Giwercman, E. Jensen, N. Hoiby, A. Kharazmi, and J. Costerton, Induction of $\beta$-lactamase production in Pseudomonas aeruginosa biofilm, Antimicrobial Agents and Chemotherapy, 35 (1991), pp. 1008-1010.

[17] I. Keren, N. Kaldalu, A. Spoering, Y. Wang, and K. Lewis, Persister cells and tolerance to antimicrobials, FEMS Microbiology Letters, 230 (2004), pp. 13-18.

[18] T. R. D. Kievit, M. D. Parkins, R. J. Gillis, R. Srikumar, H. Ceri, K. Poole, B. H. Iglewski, And D. G. Storey, Multidrug efflux pumps: Expression patterns and contribution to antibiotic resistance in Pseudomonas aeruginosa biofilms, Antimicrobial Agents and Chemotherapy, 45 (2001), pp. 1761-1770.

[19] H. Kumon, K. Tomochika, T. Matunaga, M. Ogawa, and H.Ohmori, A sandwich cup method for the penetration assay of antimicrobial agents through Pseudomonas exopolysaccharides, Microbiology Immunology, 38 (1994), pp. 615-619.

[20] H. M. Lappin-Scott and J. W. Costerton, eds., Microbial Biofilms, Cambridge University Press, Cambridge, 1995, ch. Mechanisms of the Protection of Bacterial Biofilms from Antimicrobial Agents, pp. 118-130.

[21] K. Lewis, Riddle of biofilm resistance, Antimicrobial Agents and Chemotherapy, 45 (2001), pp. 999-1007.

[22] T. Mah, B. Pitts, B. Pellock, G. Walker, P. Stewart, and G. O’Toole, A genetic basis for Pseudomonas aeruginosa biofilm antibiotic resistance, Nature, 426 (2004), pp. 306-310.

[23] M. E. Roberts and P. S. Stewart, Modeling antibiotic tolerance in biofilms by accounting for nutrient limitation, Antimicrobial Agents and Chemotherapy, 48 (2004), pp. $48-52$.

[24] — Modelling protection from antimicrobial agents in biofilms through the formation of persister cells, Microbiology, 151 (2005), pp. 75-80.

[25] S. S. Sanderson and P. S. Stewart, Evidence of bacterial adaption to monochloramine in Pseudomonas aeruginosa biofilms and evaluation of biocide action model, Biotechnology and Bioengineering, 56 (1997), pp. $201-209$. 
[26] A. Spoering And K. Lewis, Biofilms and planktonic cells of Pseudomonas aeruginosa have similar resistance to killing by antimicrobials., Journal of Bacteriology, 183 (2001), pp. 6746-6751.

[27] N. Sufya, D. Allison, And P. Gilbert, Clonal variation in maximum specific growth rate and susceptibility towards antimicrobials, Journal of Applied Microbiology, 95 (2003), pp. 1261-1267.

[28] K. D. Xu, G. A. McFeters, and P. Stewart, Biofilm resistance to antimicrobial agents, Microbiology, 146 (2000).

[29] X. Xu, P. Stewart, and X. Chen, Transport limitation of chlorine disinfection of Pseudomonas aeruginosa entrapped in alginate beads, Biotechnology and Bioengineering, 49 (1996), pp. 93-100. 